\title{
Compact MIMO Microstrip Antennas for USB Dongle Operating in 2.5-2.7 GHz Frequency Band
}

\author{
Vladimir Ssorin, Alexey Artemenko, Alexander Maltsev, \\ Alexey Sevastyanov, and Roman Maslennikov \\ Wireless Competence Center, Lobachevsky State University of Nizhny Novgorod, Gagarina Avenue 23, \\ Nizhny Novgorod 603950, Russia \\ Correspondence should be addressed to Vladimir Ssorin, vladimir.ssorin@wcc.unn.ru
}

Received 11 February 2012; Accepted 11 August 2012

Academic Editor: Hala A. Elsadek

Copyright ( $\odot 2012$ Vladimir Ssorin et al. This is an open access article distributed under the Creative Commons Attribution License, which permits unrestricted use, distribution, and reproduction in any medium, provided the original work is properly cited.

\begin{abstract}
This paper considers design of microstrip MIMO antennas for an LTE/WiMAX USB dongle operating in the 2.5-2.7 GHz frequency band. The MIMO system includes two antenna elements with an additional requirement of high isolation between them that is especially difficult to realize due to size limitations of a USB dongle. Three approaches to achieve the needed system characteristics using microstrip PCB antennas are proposed. For the first design, high port-to-port isolation is achieved by using a decoupling techniques based on a direct connection of the antenna elements. For the second approach, high port-to-port isolation of the MIMO antenna system is realized by a lumped decorrelation capacitance between antenna elements feeding points. The third proposed antenna system does not use any special techniques, and high port-to-port isolation is achieved by using only the properties of a developed printed inverted-F antenna element. The designed MIMO antenna systems have the return loss $S_{11}$ and the insertion loss $S_{21}$ bandwidths of more than $200 \mathrm{MHz}$ at the $-8 \mathrm{~dB}$ level with the correlation coefficient lower than 0.1 and exhibit pattern diversity when different antenna elements are excited. Experimental measurements of the fabricated antenna systems proved the characteristics obtained from electromagnetic simulation.
\end{abstract}

\section{Introduction}

Modern wireless communication systems should simultaneously provide high reliability and channel capacity. One of the most effective ways to achieve these goals is utilization of the multiple-input-multiple-output (MIMO) technology [1]. Antennas of a MIMO communication system consist of multiple elements and should ensure high isolation between them for efficient operation of different spacetime transmission techniques. The MIMO technology is currently used by different modern communication devices such as laptops, mobile phones, USB dongles, and others. An essential marketing trend is miniaturization of these devices that makes achieving high isolation between the antenna elements to be a challenging task due to a small (often < $0.25 \lambda_{0}$, where $\lambda_{0}$ is the operating wavelength) strictly limited area available for the antenna system. Especially, this is complicated for modern USB dongles where the total size of the device does not exceed $0.5 \lambda_{0}$. Thus, conventional diversity techniques based on different orthogonal polarizations of the antenna elements or spatial diversity with a distance between the antenna elements greater than a half of the wavelength are not always applicable for MIMO antenna systems design.

For a long time, many works were focused on developing special techniques that allow increasing the isolation between the antenna elements in a MIMO antenna system. These diversity techniques are based on different approaches: defected ground structures [2], split ring resonators [3], the electromagnetic bandgaps [4], or utilize a coupling antenna element between the antennas [5]. But application of these techniques for MIMO antenna systems requires sufficient antenna area (more than $0.25 \lambda_{0}$ ) or a very complex structure and therefore is not always suitable for the antenna systems in a modern USB dongle. Also, the isolation between the antenna elements can be increased with the help of an appropriate antenna element as, for example, a printed 
inverted-F antenna (PIFA) $[6,7]$. However, in most cases the PIFAs has a 3D structure for satisfying the compact antenna area requirement that leads to increasing a production cost.

One of the most effective approaches for development of a compact MIMO antenna system is a diversity technique based on a direct connection of the antenna elements [8-10]. A direct connection of the antenna elements allows the current induced from one antenna element to flow to the other antenna element through the connection and not to the load. The MIMO antenna system for the 2.5-2.7 GHz frequency band with the diversity technique based on direct connection of antenna elements proposed in [8] has a 3D structure, and implies that the antenna should be separately soldered to the PCB board. A 3D structure of the antenna is not cost effective and can lead to the performance degradation due to soldering misalignments in the manufacturing process. The work [9] describes a planar dual-band antenna system for the long-term evolution (LTE) communication systems in the $700 \mathrm{MHz} / 2.5-2.7 \mathrm{GHz}$ bands. The designed antenna system in [9] uses the diversity technique based on direct connection of the antenna elements for providing high port-to-port isolation in the frequency band around $700 \mathrm{MHz}$ while the diversity in the $2.5-2.7 \mathrm{GHz}$ band is achieved by conventional spatial diversity with large antenna elements separation. For the first band around $700 \mathrm{MHz}$, a relative bandwidth is only $2.5 \%$ at the $-7.4 \mathrm{~dB}$ return loss and insertion loss levels, simultaneously. The $2.5 \%$ relative bandwidth is very narrow and it is not enough for the $2.5-2.7 \mathrm{GHz}$ frequency band (more than $8 \%$ of relative bandwidth) for the considered USB dongle device.

This work aims to design a planar microstrip twoelement MIMO antenna system operating in the 2.52.7 GHz frequency band for the application in modern USB dongles for LTE or WiMAX communication systems. The common size of a modern USB dongle does not exceed $60 \mathrm{~mm} \times 20 \mathrm{~mm}$, that is, about 2 and 6 times lower in a corresponding dimension than the operating wave length $\lambda_{0}$ $(11.5 \mathrm{~cm}$ at $2.6 \mathrm{GHz})$. Besides, the antenna area should be as compact as possible (no more than $20 \times 20 \mathrm{~mm}$ ).

The current paper proposes and describes three different designs of a compact planar microstrip antenna system. All the three antennas meet the specified requirements and offer different tradeoffs between various parameters as considered below.

This paper is organized as follows. Section 2 describes the requirements that are set for a modern compact MIMO antenna system. Section 3 introduces compact two-element MIMO antenna system \#1 that uses a diversity technique based on a direct connection with a transmission line between the antenna elements [10]. Section 4 describes compact two-element MIMO antenna system \#2 that uses a diversity technique based on a lumped capacitance introduced between the antenna elements [11]. Proposed MIMO antenna system \#3 that achieves the required diversity properties without any connection of the antenna elements is described in Section 5. Finally, the conclusions are drawn in Section 6.

\section{MIMO Antenna System Requirements and Their Experimental Verification}

This section describes the requirements applying to antenna systems for correct MIMO mode operation.

The standard characteristics for MIMO antenna system analysis are scattering matrix (return loss $S_{11}$ and $S_{22}$, and insertion loss $S_{21}$ and $S_{12}$ ), diversity characteristics (envelope correlation coefficient), and radiation characteristics (radiation pattern and radiation efficiency).

A scattering matrix describes the values of the return loss $\left(S_{11}\right.$ and $\left.S_{22}\right)$ and the insertion loss $\left(S_{21}\right.$ and $\left.S_{12}\right)$ of an antenna system. In this work, the reference level both for the return loss and for the insertion loss of the MIMO antenna is defined in the required frequency bandwidth as $-8 \mathrm{~dB}$.

Diversity characteristics of a MIMO antenna system are usually described using an envelope correlation coefficient. An envelope correlation coefficient can be calculated from a $3 \mathrm{D}$ radiation pattern or a scattering matrix. The scattering matrix-based approach allows simplifying the procedure of envelope correlation coefficient calculation, that is, found from the scattering parameters as [12]:

$$
\rho_{e}=\frac{\left|S_{11}^{*} S_{12}+S_{21}^{*} S_{22}\right|^{2}}{\left(1-\left(\left|S_{11}\right|^{2}+\left|S_{21}\right|^{2}\right)\right)\left(1-\left(\left|S_{22}\right|^{2}+\left|S_{12}\right|^{2}\right)\right)} .
$$

According to the literature sources (see, e.g., [12]), the envelope correlation coefficient of less than 0.25 is recommended for efficient MIMO operation. In order to achieve the envelope correlation coefficient below 0.25 , the level of the scattering matrix coefficients with appropriate phase distribution of their complex values should be lower than $-8 \mathrm{~dB}$ as defined in the above requirements. The radiation characteristics of an antenna system are also very important. A 3D radiation pattern provides a full description about radiation characteristics including an antenna gain, a main radiation direction, and antenna efficiency. Because of the measurement complexity for the full $3 \mathrm{D}$ radiation pattern, three different orthogonal planes are often considered instead in practice.

The MIMO antenna systems designed and optimized in this work were fabricated and experimentally tested. The experimental test procedures that were used are as follows. Coaxial cables with SMA connectors were soldered directly to each antenna port to provide proper feeding of the antenna elements. It should be noted that for small antenna testing the coaxial feed cable used to connect to the measurement equipment influences on the measurement results [13]. To avoid this influence, the cables were soldered to the microstrip feed lines where the antenna elements have an $E$ field minimum [14].

The antenna return loss $\left(S_{11}\right.$ and $\left.S_{22}\right)$ and insertion loss $\left(S_{21}\right.$ and $\left.S_{12}\right)$ characteristics were measured using Agilent ENA 5071B network analyzer with a two-port calibration procedure. In addition, the soldered coaxial cables with SMA connectors were accurately deembedded in order to extract characteristics of the MIMO antenna system only. After that the envelope correlation coefficient was calculated from scattering matrix $\left(S_{11}, S_{12}, S_{21}, S_{22}\right)$. 
The radiation pattern measurements of the designed antenna systems were performed in an anechoic chamber using Agilent network analyzer N5230A. The radiation pattern was measured in three different orthogonal cut planes that are introduced relative to the spherical coordinate system shown in Figure 1. Two elevation cut planes at $\phi=$ $0^{\circ}$ and $\phi=90^{\circ}$ and one azimuth plane at $\theta=90^{\circ}$ are considered. For each cut plane, horizontally and vertically polarized components of the radiated fields were measured and then combined with the help of vector summation to find the absolute $E$-field value.

Another requirement formulated for the antennas designed in this work was to use a planar structure so that a PCB implementation was feasible for a low cost of production. An inexpensive FR4 $\left(\varepsilon_{r}=4.6\right)$ PCB material was used for the design of the MIMO antennas. The total thickness of the PCB board was $1.56 \mathrm{~mm}$, that is, a typical value for PCBs used in modern USB dongles. The PCB structure consists of four metal layers, where the thickness of the dielectric layer between the first two metal layers (used for the implementation of the microstrip line with the $50 \Omega$ characteristic impedance) is $0.2 \mathrm{~mm}$ (the width of the microstrip line is equal to $0.3 \mathrm{~mm}$ ).

\section{MIMO Antenna System Based on Direct Connection Line (\#1)}

This section describes the designed compact two-element MIMO antenna system \#1 that utilizes the diversity technique based on a direct connection line between the antenna elements [8-10].

3.1. MIMO Antenna System Description. The structure and the simulation model from CST Microwave Studio of the proposed compact two-element MIMO antenna system \#1 are shown in Figures 2(a) and 2(b), respectively.

For constructing this MIMO antenna system, a single antenna element was designed first. A monopole antenna was chosen, since a USB dongle PCB includes a large ground plane (where IC components are placed) that can be used as a monopole reference ground plane. Because of the antenna area being strictly compact, a meander line configuration of the monopole is chosen that reduces the occupied space of the antenna element to less than $0.1 \lambda_{0}$ in each dimension. Simulation results of the designed single antenna element show that it is well matched across the required $2.5-2.7 \mathrm{GHz}$ frequency band with the return loss level below $-11 \mathrm{~dB}$. After that two identical monopole antenna elements are placed near each other to form a MIMO antenna system. The total occupied antenna system area is less than $10 \mathrm{~mm} \times 20 \mathrm{~mm}$ that is $0.085 \lambda_{0} \times 0.17 \lambda_{0}$, where $\lambda_{0}$ is the wavelength in free space at the central frequency of $2.6 \mathrm{GHz}$. The distance between the two meander line monopoles was set to $6 \mathrm{~mm}$ that is about $0.05 \lambda_{0}$. In this case, the isolation between the antenna elements without applying special diversity techniques is only $2.5 \mathrm{~dB}$ that is unacceptable for the effective MIMO performance.

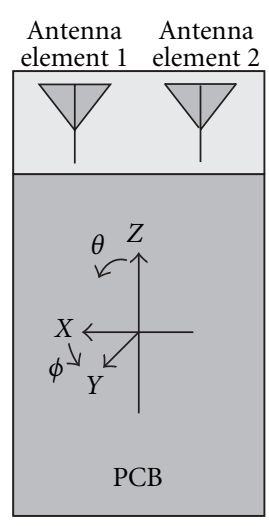

FIgURE 1: Spherical coordinate system.

In order to improve the MIMO characteristics, the monopole antennas were connected to each other by a connection line in low impedance connection points of the monopoles. The main concept of this technology is described in Figure 3 which shows two parallel dipole antennas (for simplicity). In Figure 3(a), two separate antennas are shown without any connection between each other. Some part of the signal being transmitted by one of the dipoles will be coupled directly into the neighboring dipole that leads to poor isolation between them. Figure 3(b) illustrates antenna system comprised of two closely separated dipoles with connection elements between them. In this case, the current induced from one antenna element flows through the connection to the other antenna element and does not pass through the load. Thus, both antennas form a radiation pattern with some dedicated direction of main radiation. When another antenna element is excited, the radiation pattern of the MIMO antenna system has an opposite direction of main radiation. It provides the pattern diversity when different antenna elements are excited or, equivalently, high port-to-port isolation is achieved.

The direct connection line provides high port-to-port isolation at some resonant frequency that was found to be slightly lower than the central radiation bandwidth frequency. This effect is due to an additional electrical length of the connection line that increases the electrical length of the antenna elements. Moreover, at this case, it was observed that the resonance of the antenna elements return loss $\left(S_{11}\right.$ and $S_{22}$ ) is shifted from the required $2.5-2.7 \mathrm{GHz}$ frequency band to higher frequency $(2.6-2.8 \mathrm{GHz})$, that leads to the $-2.5 \mathrm{~dB}$ return loss level in the needed band. In order to adjust the resonant bandwidth of the return loss and the insertion loss to the required $2.5-2.7 \mathrm{GHz}$ frequency band, the matching circuit consisting of lumped inductors $\left(L_{1}\right)$ connected in series to each of the antenna elements is introduced into the antenna structure (see Figure 2(a)).

It was observed that the usage of the direct connection line and the matching circuit lead to return loss bandwidth shrinkage comparing to the case without the diversity technique implementation (without direct connection line). However, the bandwidth shrinkage depends on the connection line parameters. Hence, the optimization of the 


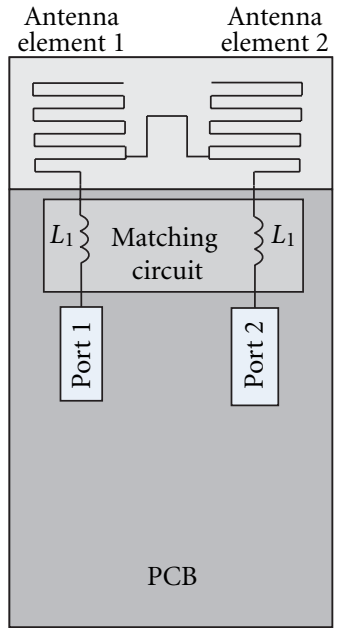

(a)

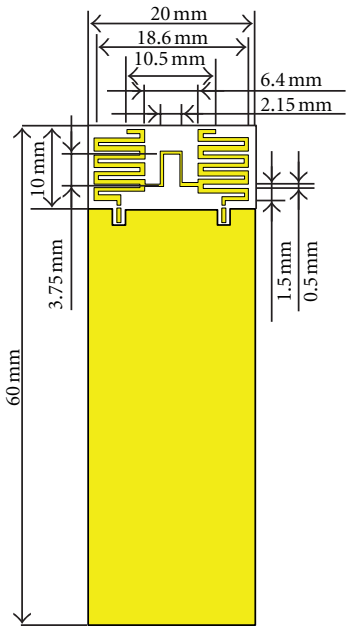

(b)
Figure 2: Structure (a) and CST Microwave Studio model (b) of the designed compact two-element MIMO antenna system \#1.

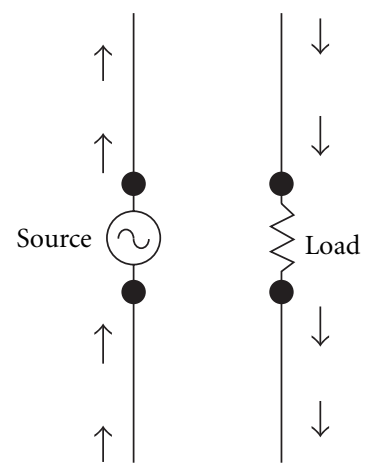

(a) (b)

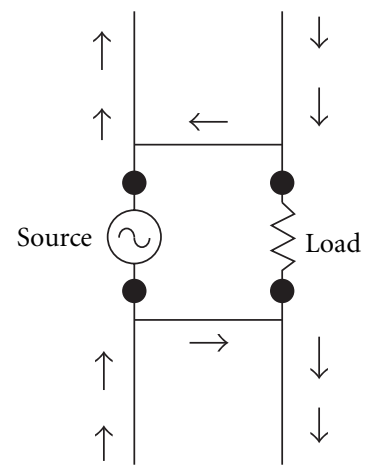

Figure 3: Antenna elements without (a) and with (b) direct connection lines.

two-element MIMO antenna system structure should be performed in order to provide the maximum achievable bandwidth of the return loss $\left(S_{11}\right.$ and $\left.S_{22}\right)$ and the insertion loss $\left(S_{21}\right.$ and $\left.S_{12}\right)$ simultaneously.

The bandwidth optimization was performed by variation of the connection line parameters: length, width, and connection point position. Preliminary optimization revealed that the maximum impact on the antenna bandwidth has the connection line length (viz. $L$ parameter) that is shown in Figure 4.

Thus, in the final optimization process, the length of the connection line was varied while the width and the connection points positions were fixed after the preliminary optimization. The variation range of the connection line length $L$ for the optimization was chosen from 1 to $6 \mathrm{~mm}$. During the optimization of the connection line length, the resonant frequency of $S_{21}$ and $S_{11}$ was kept equal to $2.6 \mathrm{GHz}$. This was done by adjusting of the meander line length and the matching circuit inductance values.

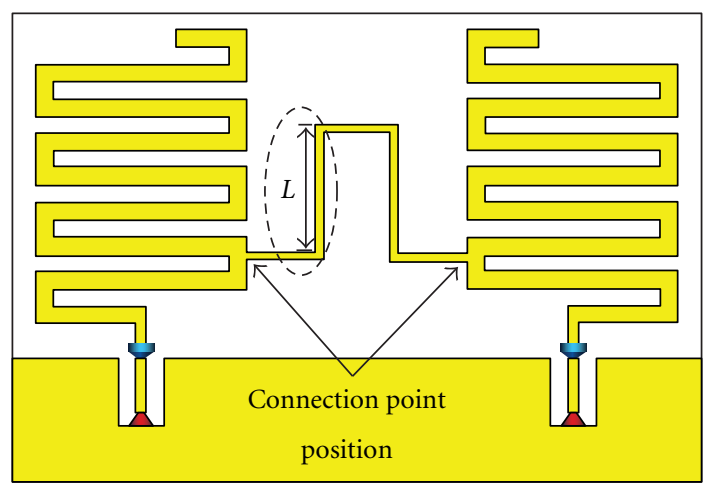

FIGURE 4: Simulation model with varied connection line length $L$ of the MIMO antenna system \#1.

Table 1 shows the return loss $\left(S_{11}\right.$ and $\left.S_{22}\right)$ and the insertion loss $\left(S_{12}\right.$ and $\left.S_{21}\right)$ bandwidths around the central frequency of $2.6 \mathrm{GHz}$ for different connection line lengths. The bandwidths were estimated at the $-8 \mathrm{~dB}$ level of $S_{11}$ and $S_{22}$ and at the $-8 \mathrm{~dB}$ level of $S_{12}$ and $S_{21}$.

The results presented in Table 1 show that the return loss bandwidth decreases and the insertion loss bandwidth increases while increasing the connection line length. Also, it is observed that the optimum value of the connection line length $L$, which corresponds to the equal values of the $S_{21}$ and $S_{11}$ bandwidths of about $210 \mathrm{MHz}$, is equal to $4 \mathrm{~mm}$. For all other values of the connection line length, either the return or the insertion loss bandwidths are smaller than $210 \mathrm{MHz}$. Note, that the optimal MIMO antenna system parameters were achieved with the matching circuit consisting of a lumped inductance $L_{1}=4.5 \mathrm{nH}$.

3.2. Simulation and Measurement Results. The MIMO antenna system with the optimal connection line parameters was fabricated and tested. A photo of the fabricated MIMO antenna system with assembled coaxial cables is shown in Figure 5.

The measured and simulated S-parameters for the optimized MIMO antenna system are shown in Figure 6. Good correspondence between the measurement and simulation results is observed. The measurement results indicate that the return loss $S_{11}$ and the insertion loss $S_{21}$ of the fabricated MIMO antenna system are below $-8.5 \mathrm{~dB}$ levels in the required $2.5-2.7 \mathrm{GHz}$ frequency band.

The measured and simulated results for the envelope correlation coefficient are shown in Figure 7. It may be seen that the measured and simulated envelope correlation coefficients are lower than 0.16 across the required bandwidth of 2.52.7 GHz that provides effective MIMO operation.

Figure 8 presents measured and simulated absolute values of the radiation pattern cuts for each antenna port in all the three planes at $2.6 \mathrm{GHz}$. During the measurements, one of the two-ports was excited, while the other was terminated with a $50 \Omega$ matching load. Thus, two different radiation patterns were obtained-one for each of the ports.

The measurement and simulation results show that the designed MIMO antenna system has pattern diversity at both 
TABLE 1: $S_{11}$ and $S_{21}$ bandwidths for different $L$.

\begin{tabular}{lcc}
\hline$L$ value, $\mathrm{mm}$ & $\begin{array}{c}S_{11} \text { and } S_{22} \text { bandwidth, } \\
\mathrm{MHz}\end{array}$ & $\begin{array}{c}S_{12} \text { and } S_{21} \text { bandwidth, } \\
\mathrm{MHz}\end{array}$ \\
\hline 1 & 226 & 130 \\
2 & 221 & 146 \\
3 & 216 & 173 \\
4 & 209 & 207 \\
5 & 196 & 425 \\
6 & 184 & 435 \\
\hline
\end{tabular}

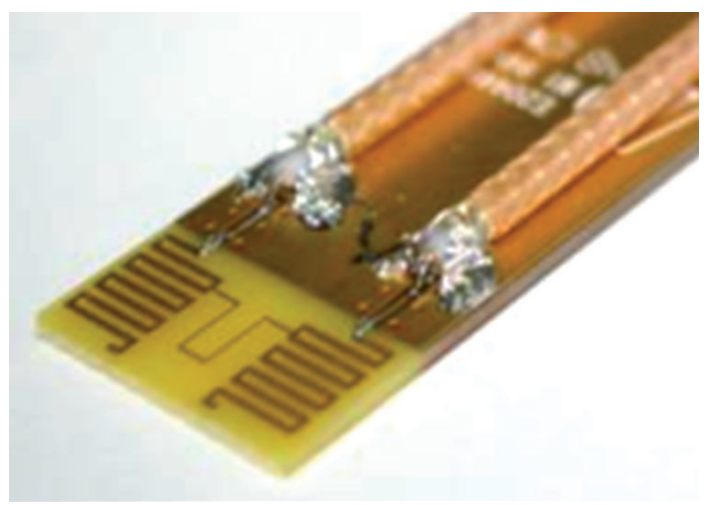

Figure 5: Photo of the fabricated MIMO antenna system \#1 with assembled cables.

azimuth cut $\theta=90^{\circ}$ (Figure 8(c)) and elevation cut $\phi=0^{\circ}$ (Figure 8(a)) that proves the effectiveness of the diversity technique based on a direct connection of the antenna elements. At the elevation cut $\phi=90^{\circ}$ (Figure 8(b)) almost an omnidirectional radiation pattern is observed as expected since the antenna elements are placed symmetrically to this cut plane. The maximum measured gain of the MIMO antenna system at the $2.6 \mathrm{GHz}$ frequency is $1.5 \mathrm{dBi}$.

The measured characteristics satisfy all the requirements that were set to the MIMO antenna system of a modern USB dongle.

\section{MIMO Antenna System Based on a Lumped Decorrelation Capacitance (\#2)}

This section describes the second designed compact twoelement MIMO antenna system that uses the diversity technique utilizing a lumped capacitance introduced between the antenna elements [11].

4.1. MIMO Antenna System Description. The structure and the simulation model from CST microwave studio of the MIMO antenna system \#2 are shown in Figures 9(a) and 9(b), respectively.

Similar to the first MIMO antenna system, a monopole antenna with a modified configuration was used as an antenna element of the second antenna system. A triangular antenna element configuration allows reducing its occupied space to less than $0.1 \lambda_{0}$ in each dimension. Simulation results

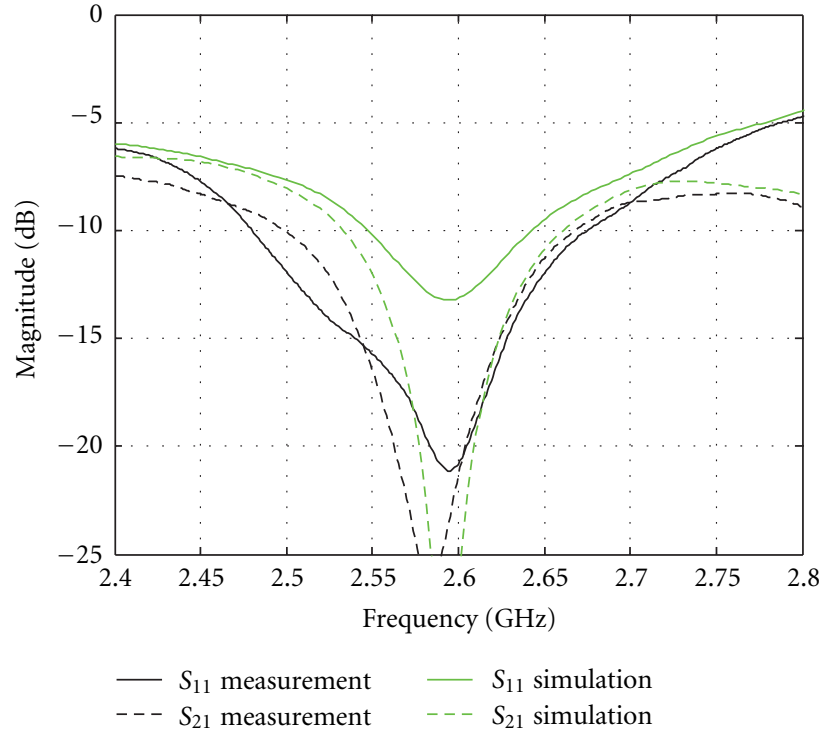

FIGURE 6: $S$-parameters for the optimized structure of the MIMO antenna system \#1 (measurements versus simulations).

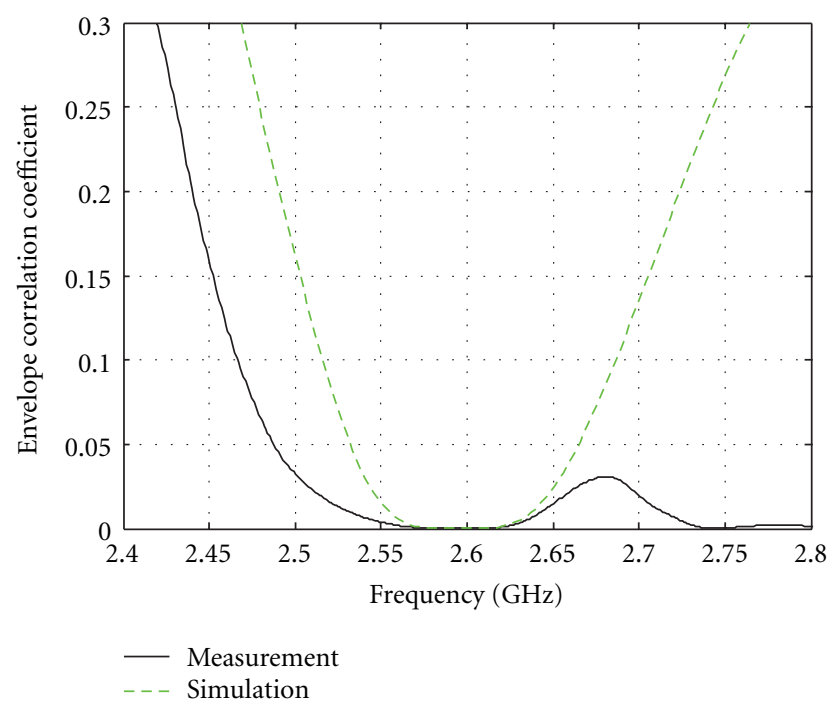

FIGURE 7: Envelope correlation coefficient of the MIMO antenna system \#1 (measurements versus simulations).

of the designed single antenna element show that it is well matched with the return loss level below $-15 \mathrm{~dB}$ across the required $2.5-2.7 \mathrm{GHz}$ frequency band. Two identical monopole antennas occupy area less than $10 \mathrm{~mm} \times 20 \mathrm{~mm}$ with $5.5 \mathrm{~mm}\left(0.048 \lambda_{0}\right)$ distance between them. However, such antenna system configuration without additional diversity techniques provides only $3 \mathrm{~dB}$ isolation between the antenna elements.

In order to improve isolation between the antenna elements a lumped decorrelation capacitance is mounted between the antenna elements feeding points. The concept of this technology is the same as for the diversity technique 


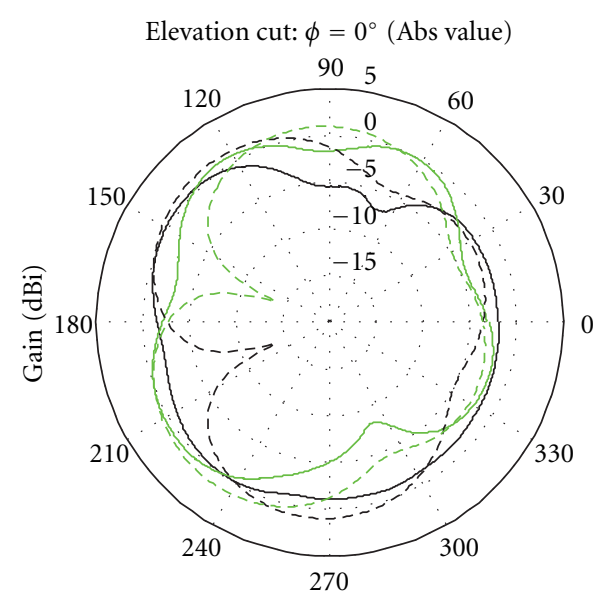

$\begin{array}{ll}\text { Measurement port } 1 & --- \text { Simulation port } 1 \\ \text { Measurement port } 2 & --- \text { Simulation port } 2\end{array}$

(a)

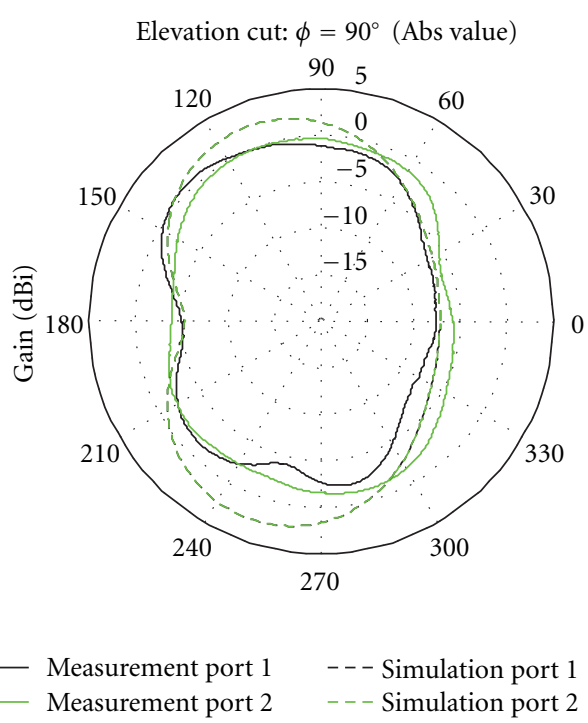

(b)

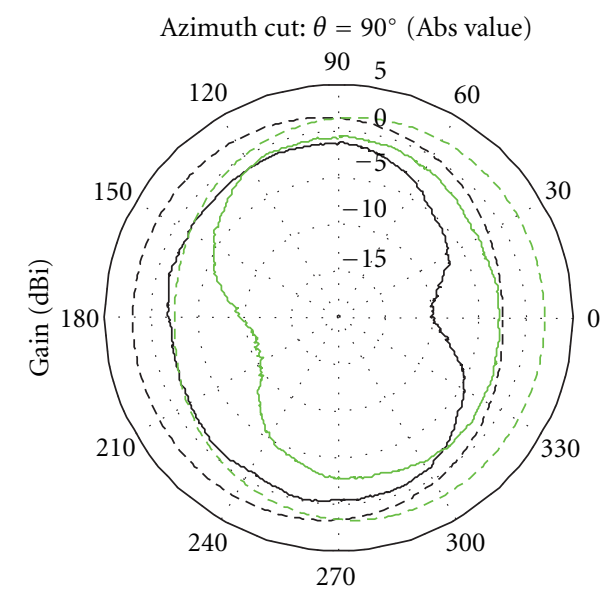

$\begin{array}{ll}\text { Measurement port } 1 & --- \text { Simulation port } 1 \\ \text { Measurement port } 2 & --- \text { Simulation port } 2\end{array}$

(c)

FIGURE 8: Radiation pattern cuts of the MIMO antenna system \#1 (measurements versus simulations).

based on a direct connection line that is specified in the previous section (Section 3 ). The main difference between these two approaches consist in usage of a lumped decorrelation capacitance instead of a direct connection line.

The lumped decorrelation capacitance allows to tune the antenna coupling so that to provide high isolation. The capacitance value is optimized to achieve the low insertion loss $S_{21}$ and $S_{12}$ (high isolation between the antenna elements) in the required frequency band. It was found by adjusting the capacitance value that the $0.8 \mathrm{pF}$ capacitance provides the highest isolation at the $2.5-2.7 \mathrm{GHz}$ frequency band given that the geometry of the antenna elements is fixed. The usage of the capacitor with $0.8 \mathrm{pF}$ allows improving the isolation from $3 \mathrm{~dB}$ to $10 \mathrm{~dB}$ across the required frequency band. It was observed that the usage of the decorrelation capacitance leads to variations of the operational frequency band of the return loss $S_{11}$ and $S_{22}$ (the return loss level changes from $-15 \mathrm{~dB}$ to $-2.5 \mathrm{~dB}$ in the 2.5-2.7 GHz frequency band). In order to simultaneously adjust the return loss and the insertion loss to the same resonant frequency, a matching circuit consisting of two lumped inductors connected in shunt $\left(L_{2}\right)$ and in series $\left(L_{1}\right)$ to each of the antenna elements is introduced into the antenna structure (see Figure 9(a)). Since the isolation level is very high, an additional impedance network does not change the isolation. To adjust the return loss $S_{11}$ to the required frequency band, the inductors values $L_{1}=3 \mathrm{nH}$ and $L_{2}=4.6 \mathrm{nH}$ are used.

The final results for the second designed MIMO antenna system with the lumped capacitance and the matching circuit 


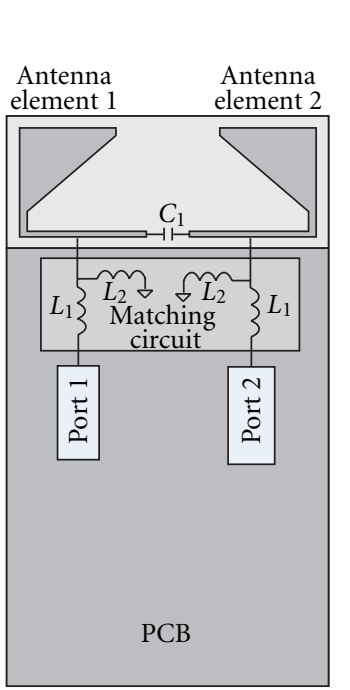

(a)

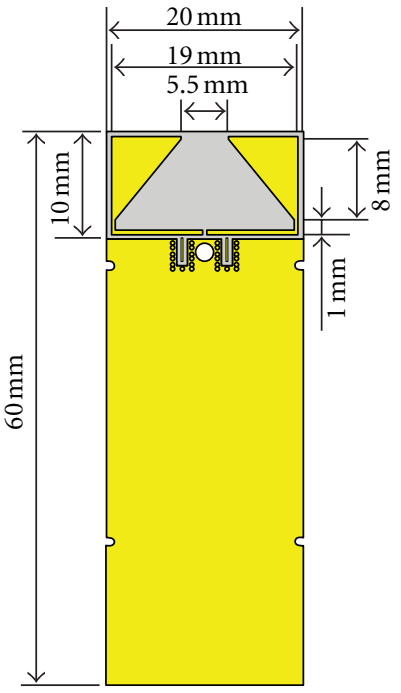

(b)
FIGURE 9: Structure (a) and CST Microwave Studio model (b) of the designed compact two-element MIMO antenna system \#2.

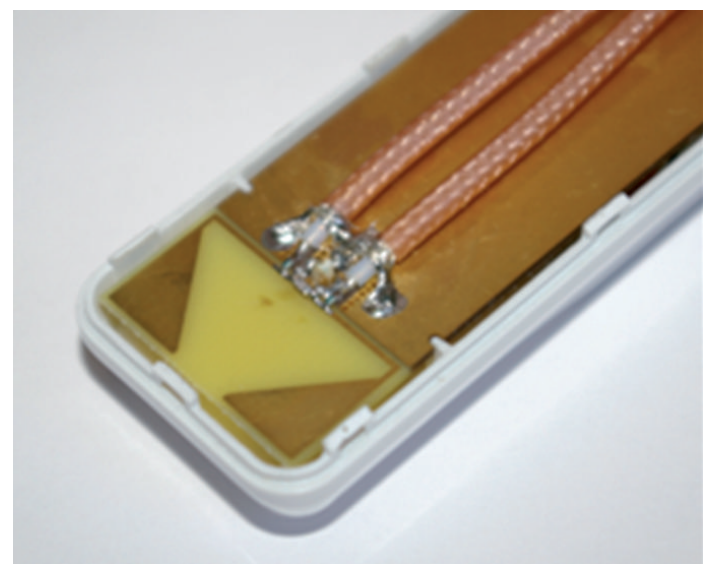

Figure 10: Photo of the fabricated MIMO antenna system \#2 assembled with the cables.

indicate that the return loss $\left(S_{11}\right.$ and $\left.S_{22}\right)$ and the insertion loss $\left(S_{21}\right.$ and $\left.S_{12}\right)$ have $-8 \mathrm{~dB}$ levels in the required 2.5$2.7 \mathrm{GHz}$ frequency band.

4.2. Simulation and Measurement Results. The MIMO antenna system \#2 that uses the diversity technique utilizing a lumped capacitance was fabricated and tested. A photo of the fabricated MIMO antenna system \#2 assembled with the coaxial cables and lumped elements is shown in Figure 10.

The measured and simulated return loss $\left(S_{11}\right)$ and insertion loss $\left(S_{21}\right)$ are shown in Figure 11. The demonstrated results show a good agreement between the measurements and simulations. The measurement results indicate that the fabricated MIMO antenna system can operate in the 2.5$2.7 \mathrm{GHz}$ frequency band with at least the $-8 \mathrm{~dB}$ level of the return loss $S_{11}$ and high isolation between the antenna elements (the insertion loss $S_{21}$ is below $-8.5 \mathrm{~dB}$ ).

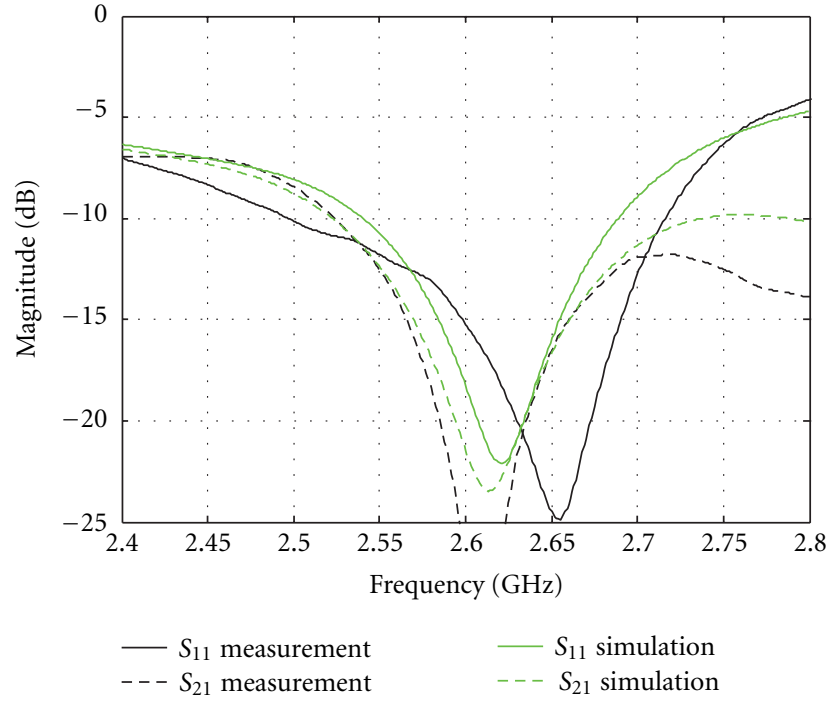

FIGURE 11: S-parameters of the MIMO antenna system \#2 (measurements versus simulations).

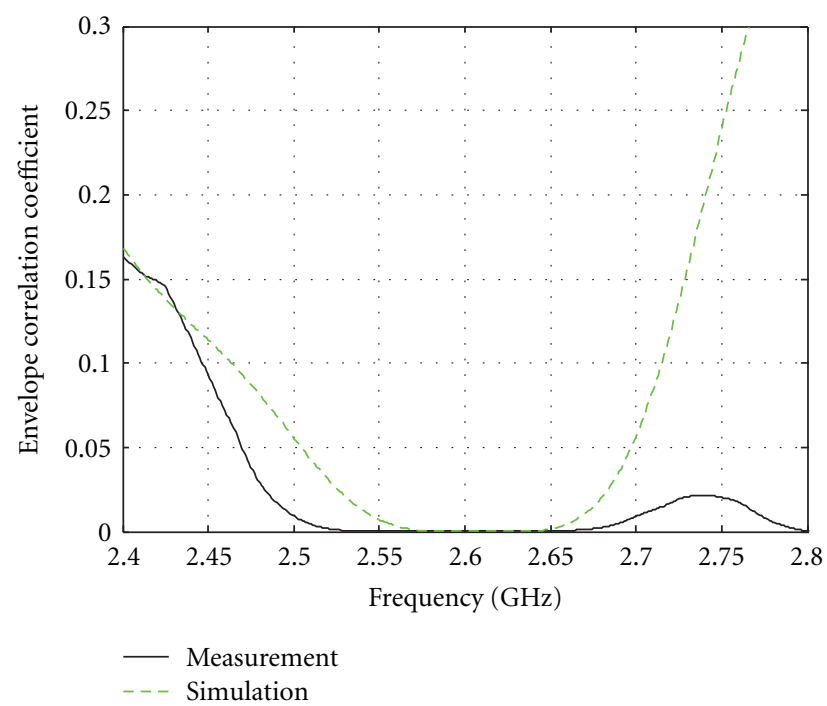

FIGURE 12: Envelope correlation coefficient of the MIMO antenna system \#2 (measurements versus simulations).

The measured and simulated results for the envelope correlation coefficient are shown in Figure 12. It may be seen that the measured and simulated envelope correlation coefficients are lower than 0.06 across the required bandwidth of 2.5-2.7 GHz.

Figure 13 presents measured and simulated absolute values of the radiation pattern cuts for each antenna port in all the three planes at $2.6 \mathrm{GHz}$. During the measurements, one of the two-ports was excited, while the other was terminated with a $50 \Omega$ matching load. Thus, two different radiation patterns were obtained-one for each of the ports.

The measurement and simulation results show the radiation properties similar to the MIMO antenna system \#1. 

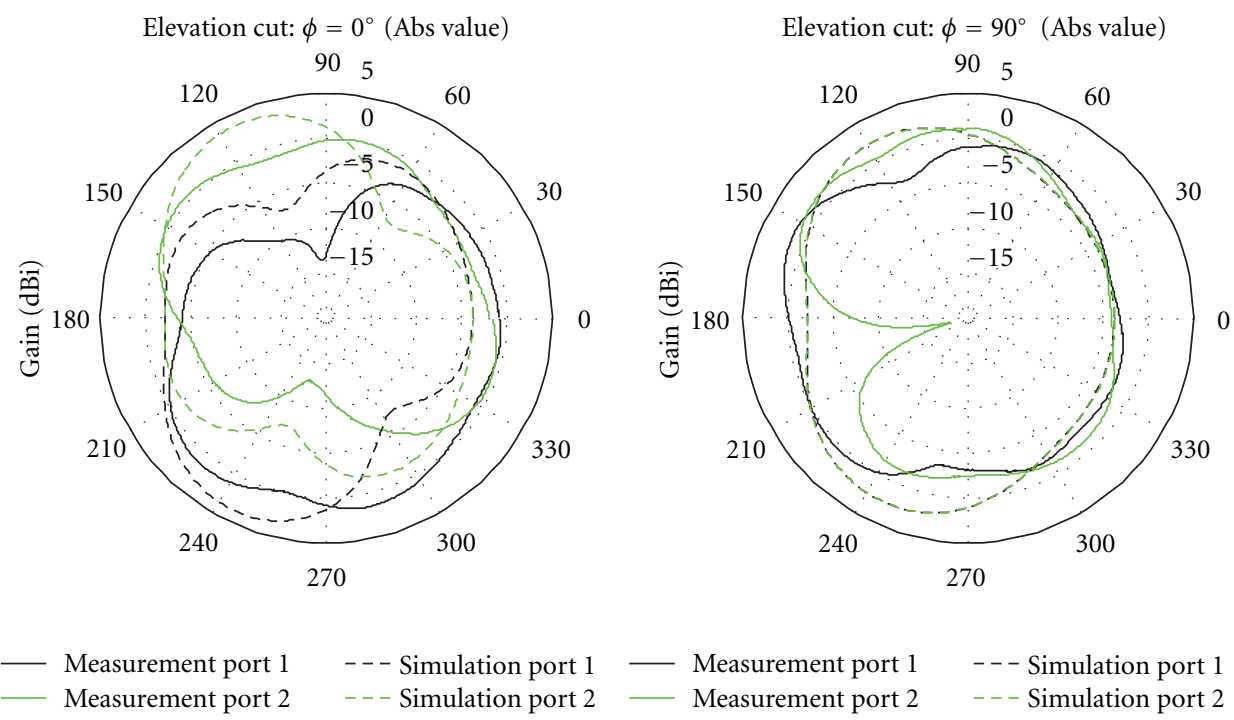

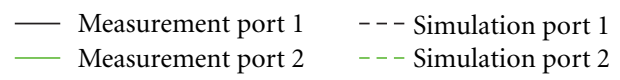

(a) (b)

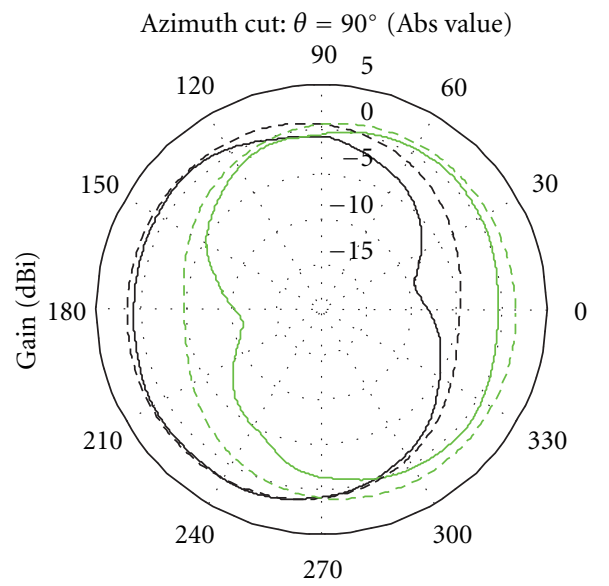

$\begin{array}{ll}\text { Measurement port } 1 & --- \text { Simulation port } 1 \\ \text { Measurement port } 2 & --- \text { Simulation port } 2\end{array}$

(c)

Figure 13: Radiation pattern cuts of the MIMO antenna system \#2 (measurements versus simulations).

More specifically, the second MIMO antenna system has pattern diversity at the azimuth cut $\theta=90^{\circ}$ (Figure 13(c)) and the elevation cut $\phi=0^{\circ}$ (Figure 13(a)) that proves the effectiveness of the proposed MIMO antenna design approach. At the elevation cut $\phi=90^{\circ}$ (Figure 13(b)), almost an omnidirectional radiation pattern is observed as expected, since the antenna elements are placed symmetrically to this cut plane. The maximum measured gain of the MIMO antenna system at the $2.6 \mathrm{GHz}$ frequency is $2.5 \mathrm{dBi}$.

Comparison of the measurement and simulation results shows their good agreement. Hence, the obtained results prove sufficient return loss bandwidth with high port-toport isolation and confirm pattern diversity of the designed compact MIMO antenna system \#2. As for the first design, all the set requirements are met.

\section{MIMO Antenna System Using PIFA Elements (\#3)}

This section describes the third designed compact twoelement MIMO antenna system (\#3). To achieve sufficient isolation between the antenna elements, this antenna system uses only antenna element properties and does not use special diversity techniques which application may be a subject of different limitations arising, for example, from the intellectual property rights.

5.1. MIMO Antenna System Description. The structure and the simulation model from CST Microwave Studio of the designed MIMO antenna system \#3 are shown in Figures $14(\mathrm{a})$ and 14(b), respectively. 
For constructing a MIMO antenna system, a planar inverted- $F$ antenna (PIFA) was used. A PIFA is another type of a monopole antenna which allows reducing the monopole dimensions. It is achieved by using a folded stub of one part of a monopole to the ground plane. One of the inherent properties of the PIFA antenna is that its radiation is located mostly in one-half space. Therefore, two closely separated PIFAs with opposite symmetrical (mirror) orientations provide pattern and polarization diversity. In this work, it was found that two closely spaced PIFAs in strictly compact antenna area (as in USB dongle) allow achieving the convenient isolation between elements of MIMO antenna system without using the special diversity techniques.

In order to construct a MIMO antenna system, two PIFA elements were symmetrically located along the edges of the USB PCB as shown in Figure 14. To achieve the required antenna elements isolation, the distance between them was adjusted. It was observed that with an increase of the distance between the elements (i.e., ground plane size expansion), the isolation between the antenna elements increases. It was observed that the optimum distance between two PIFAs, which corresponds to the isolation level not less than $8 \mathrm{~dB}$ and return loss level not more than $-8 \mathrm{~dB}$ across the required 2.5-2.7 GHz band, is equal to $12 \mathrm{~mm}$ or $0.1 \lambda_{0}$. Therefore, the total occupied antenna system area is less than $21.5 \mathrm{~mm}$ $\times 20 \mathrm{~mm}$ that is equivalent to $0.19 \lambda_{0} \times 0.18 \lambda_{0}$. However, it should be noted that the ground plane (with the $21.5 \mathrm{~mm} \times$ $12 \mathrm{~mm}$ size) between two PIFAs can be used for mounting additional IC components that further improves the PCB area utilization.

5.2. Simulation and Measurement Results. The MIMO antenna system \#3 that uses PIFA elements was fabricated and tested. A photo of the fabricated MIMO antenna system \#3 assembled with the coaxial cables is shown in Figure 15.

The measurement and simulation results for the return loss $\left(S_{11}\right)$ and the insertion loss $\left(S_{21}\right)$ are plotted in Figure 11. The demonstrated results show a good agreement between the measurements and simulations. The measurement results indicate that the fabricated MIMO antenna system can operate in the $2.5-2.7 \mathrm{GHz}$ frequency band with at least the $-8 \mathrm{~dB}$ level of the return loss $S_{11}$ and high isolation between the antenna elements (the insertion loss $S_{21}$ is below $-8.5 \mathrm{~dB})$. Note that the minimum isolation $(8.5 \mathrm{~dB})$ for the mirror symmetrical orientation of the two PIFAs is much better than in the case of other antenna types (like monopoles) that are located near to each other without applying any additional diversity techniques. In case when two parallel monopoles are used only $3.5 \mathrm{~dB}$ isolation can be achieved.

Figure 17 presents the measured and simulated results for the envelope correlation coefficient. It may be seen that the measured and simulated envelope correlation coefficients are lower than 0.1 across the required bandwidth of 2.5-2.7 GHz.

The measured and simulated absolute values of the radiation pattern cuts for each antenna port are shown in Figure 18 for all the three planes at $2.6 \mathrm{GHz}$. During the

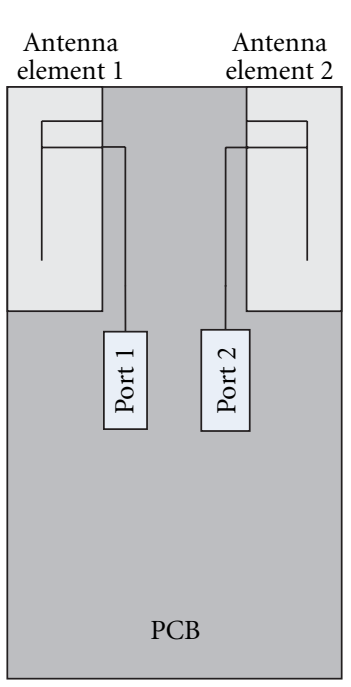

(a)

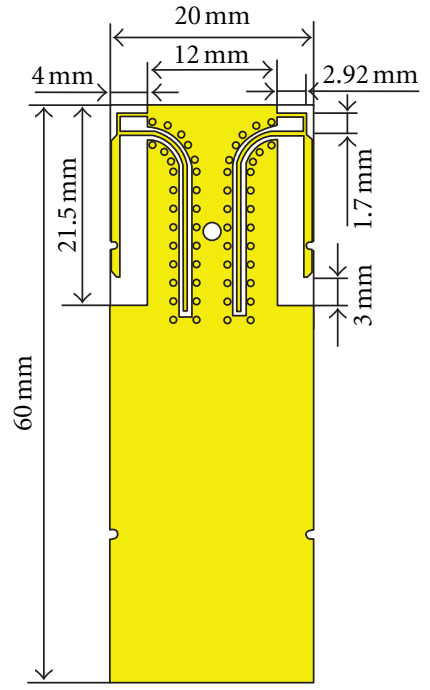

(b)
FIGURE 14: Structure (a) and CST Microwave Studio model (b) of the designed compact two-element MIMO antenna system \#3.

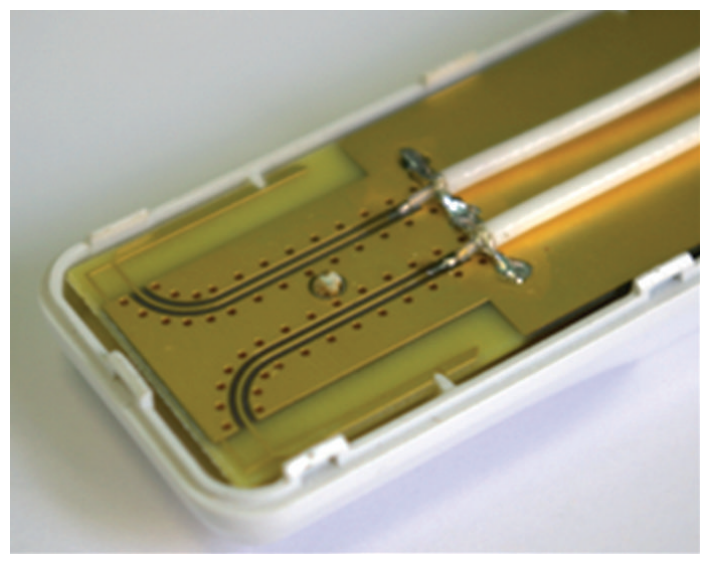

Figure 15: Photo of the fabricated MIMO antenna system \#3 with the assembled cables.

measurements, one of the two-ports was excited, while the other was terminated with a $50 \Omega$ matching load. Thus, two different radiation patterns were obtained-one for each of the ports.

The measurement and simulation results show that the designed antenna system has an almost omnidirectional radiation pattern at the elevation cut $\phi=90^{\circ}$ (Figure 18(b)) and pattern diversity at both azimuth cut $\theta=90^{\circ}$ (Figure 18(c)) and the elevation cut $\phi=0^{\circ}$ (Figure 18(b)). The radiation pattern diversity is achieved due to the inherent properties of the PIFA antenna. The maximum measured gain of the MIMO antenna system at the $2.6 \mathrm{GHz}$ frequency is $2.5 \mathrm{dBi}$.

The measurements results satisfy all characteristics requirements that are applied to MIMO antenna system in modern USB dongles. 


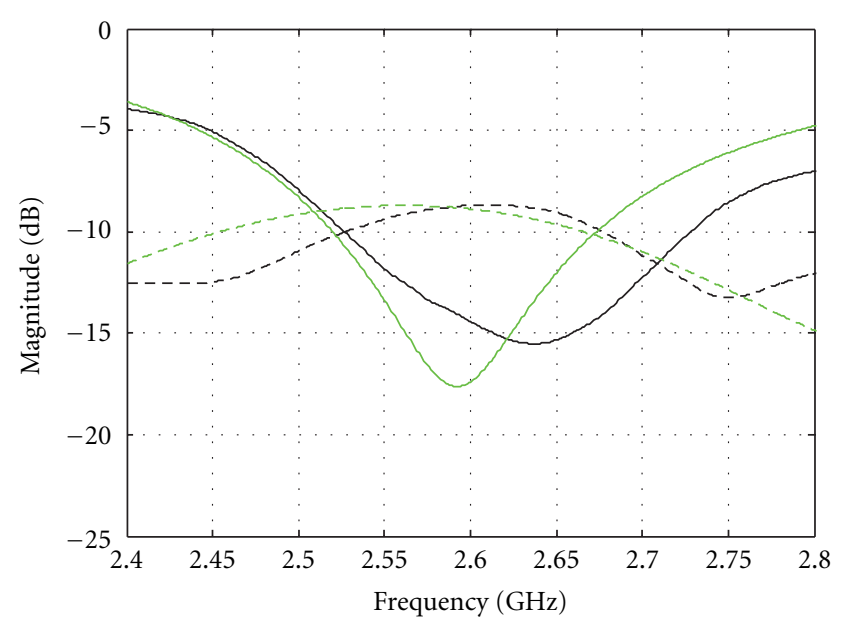

$\begin{array}{ll}-S_{11} \text { measurement } & -S_{11} \text { simulation } \\ ---S_{21} \text { measurement } & ---S_{21} \text { simulation }\end{array}$

Figure 16: S-parameters of the MIMO antenna system of the MIMO antenna system \#3 (measurements versus simulations).

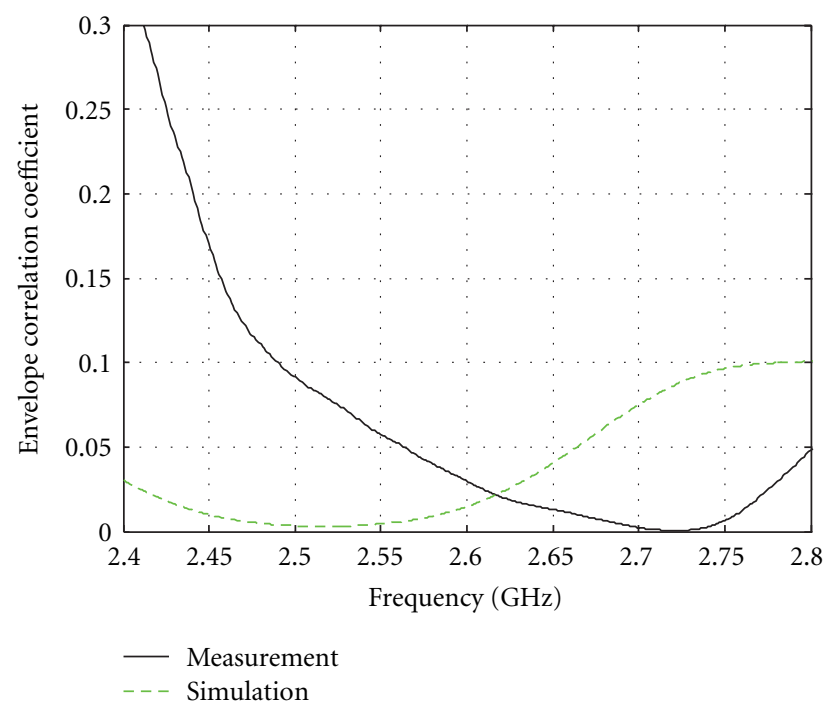

FIGURE 17: Envelope correlation coefficient of the MIMO antenna system \#3 (measurements versus simulations).

\section{MIMO Antenna Systems Comparison}

All the three MIMO antennas systems presented in this paper achieve the characteristics required for an efficient MIMO system operation in a USB dongle as formulated in the beginning of this work.

Despite many similarities, quite important differences exist between the three designs that may impact one or another design selection in a particular case.

All the designed MIMO antennas have planar microstrip structures and use a standard PCB technology that provides a low production cost. First two antenna systems occupy only $10 \mathrm{~mm} \times 20 \mathrm{~mm}$ area that is equivalent to 0.085 $\lambda_{0} \times 0.17 \lambda_{0}$. The antenna system \#3 needs a larger area $\left(21.5 \mathrm{~mm} \times 20 \mathrm{~mm}\right.$ or $0.19 \lambda_{0} \times 0.18 \lambda_{0}$. $)$ but keeps some space in between the PIFA elements for mounting of the IC components.

Another important differentiation is that MIMO antenna systems \#1 and \#2 use a special diversity technique by connecting the antenna elements while the MIMO antenna system \#3 utilizes independent antenna elements (Figure 16). For MIMO antenna system \#1, a direct connection line between the antenna elements was used. The geometry of the connection line allows adjusting the insertion loss $S_{21}$ resonance to the required frequency. Hence, the final MIMO antenna system optimization should be carried out before its fabrication. In MIMO antenna system \#2, the high port-toport isolation is achieved using the same concept but instead of a direct connection line it uses a lumped capacitance introduced between the antenna elements. The introduction of the capacitor allows carrying out the final MIMO antenna system optimization after its fabrication by changing the capacitance value.

Regarding the bandwidth of the antenna system, all the three designs presented in this work achieve the formulated required characteristics for efficient MIMO system operation, namely at least $-8 \mathrm{~dB}$ level of the return loss $S_{11}$ and high isolation between the antenna elements (the insertion loss $S_{21}$ below $-8.5 \mathrm{~dB}$ ) across the required $2.5-2.7 \mathrm{GHz}$ frequency band. However, the bandwidth requirements are met only marginally for all the three designs. The bandwidth for MIMO antenna systems \#1 and \#2 is limited because of application of the direct connection diversity techniquethe direct connection line and the lumped capacitance significantly reduce the antenna system bandwidth relative to a single element case. The value of the bandwidth for the MIMO antenna system \#3 is limited by a small size of the ground plane between the antenna elements. In order to achieve the required $200 \mathrm{MHz}$ bandwidth, the rigorous parameters optimization was done for all the three MIMO antenna systems and optimal parameters were selected.

None of the three proposed systems is considered to be a preferred solution and that was one of the motivations for their joint presentation in this work. However, an introduction of new additional system constraints may shift the designer's preference towards one of the approaches.

\section{Conclusion}

This work presented the three different microstrip MIMO antenna system designs for LTE/WiMAX USB dongle applications operating in the $2.5-2.7 \mathrm{GHz}$ frequency band. These antenna systems include two antenna elements with high isolation between them and implemented in a strictly compact area. For the first antenna system design, high port-toport isolation is achieved by using a decoupling techniques based on a direct connection of the antenna elements. For the second approach, high port-to-port isolation for the MIMO antenna system is realized by a lumped decorrelation capacitance between antenna elements feeding points. The 

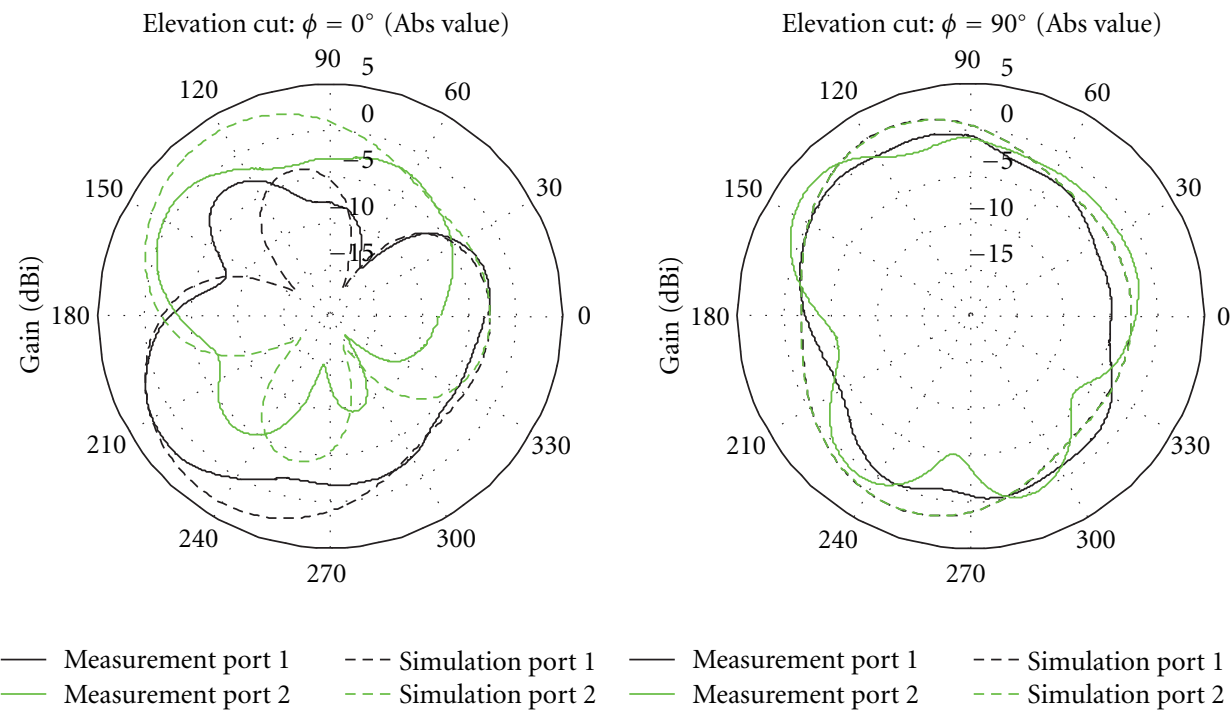

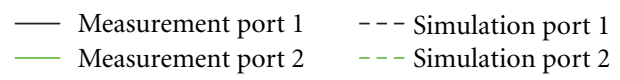

(a) (b)

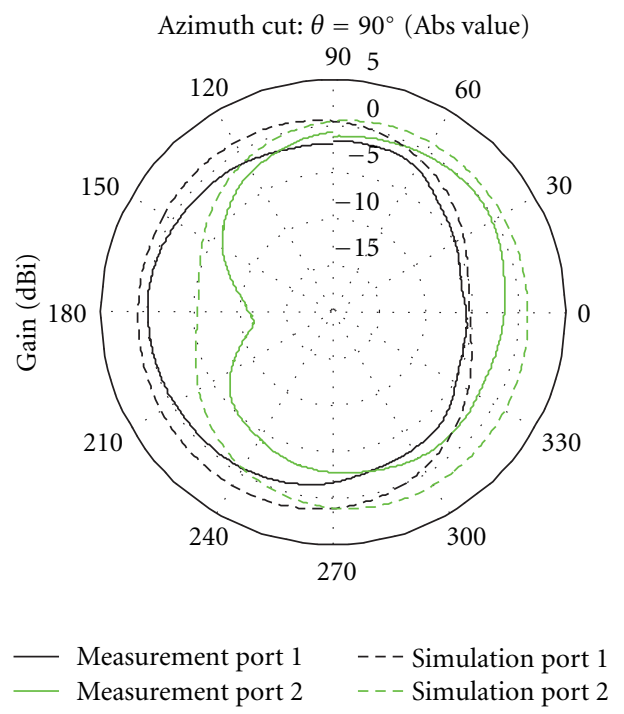

(c)

FIGURE 18: Radiation pattern cuts of the MIMO antenna system \#3 (measurements versus simulations).

third proposed antenna system does not use any special techniques, and high port-to-port isolation is achieved by using only the properties of a developed printed inverted$\mathrm{F}$ antenna element. The proposed MIMO antenna systems were designed in the low-cost FR4 PCB technology that is typically used for a USB dongle realization.

The performed simulation and experimental investigations of the S-parameters, envelope correlation coefficient, and radiation patterns for the designed MIMO antenna systems demonstrate low return loss $(<-8 \mathrm{~dB})$ and high port-to-port isolation $(>8.5 \mathrm{~dB})$ in the required frequency band of 2.5-2.7 GHz and confirm their pattern diversity. The designed MIMO antenna systems can be used in different 2.5-2.7 GHz applications of USB dongles like LTE and WiMAX where effective support for MIMO transmission is required.

\section{References}

[1] A. J. Paulraj, D. A. Gore, R. U. Nabar, and H. Bölcskei, "An overview of MIMO communications-a key to gigabit wireless," Proceedings of the IEEE, vol. 92, no. 2, pp. 198-218, 2004.

[2] M. Karakoikis, C. Soras, G. Tsachtsiris, and V. Makios, "Compact dual-printed inverted-F antenna diversity systems for portable wireless devices," IEEE Antennas and Wireless Propagation Letters, vol. 3, no. 1, pp. 9-14, 2004.

[3] H. Lihao, Z. Huiling, H. Zhang, and C. Quanming, "Reduction of mutual coupling between closely-packed antenna elements with split ring resonator (SRR)," in Proceedings of the International Conference on Microwave and Millimeter Wave Technology (ICMMT '10), pp. 1873-1875, Chengdu, China, May 2010.

[4] F. Yang and Y. Rahmat-Samii, "Microstrip antennas integrated with electromagnetic band-gap (EBG) structures: a 
low mutual coupling design for array applications," IEEE Transactions on Antennas and Propagation, vol. 51, no. 10, pp. 2936-2946, 2003.

[5] A. C. K. Mak, C. R. Rowell, and R. D. Murch, "Isolation enhancement between two closely packed antennas," IEEE Transactions on Antennas and Propagation, vol. 56, no. 11, pp. 3411-3419, 2008.

[6] S. Zhang, S. N. Khan, and S. He, "Reducing mutual coupling for an extremely closely-packed tunable dual-element PIFA array through a resonant slot antenna formed in-between," IEEE Transactions on Antennas and Propagation, vol. 58, no. 8, pp. 2771-2776, 2010.

[7] Q. Wang, H. Zhang, D. Plettemeier, E. Ohlmer, and G. Fettweis, "Design and performance evaluation of handset MIMO antenna prototypes," in Proceedings of the International ITG Workshop on Smart Antennas (WSA '10), pp. 375-382, Bremen, Germany, February 2010.

[8] F. M. Caimi and M. Mongomery, "Dual feed, single element antenna for WiMAX MIMO application," Internal Journal of Antennas and Propagation, vol. 2008, Article ID 219838, 5 pages, 2008.

[9] I. Dioum, A. Diallo, C. Luxey, and S. M. Farsi, "Dual-band monopole MIMO antennas for LTE mobile phones," in Proceedings of the 20th International Conference on Applied Electromagnetics and Communications (ICECom '10), Dubrovnik, Croatia, September 2010.

[10] V. Ssorin, A. Artemenko, A. Sevastyanov, and R. Maslennikov, "Compact bandwidth-optimized two element MIMO antenna system for 2.5-2.7 GHz band," in Proceedings of the 5th European Conference on Antennas and Propagation (EUCAP '11), pp. 319-323, Rome, Italy, April 2011.

[11] V. Ssorin, A. Artemenko, A. Sevastyanov, and R. Maslennikov, "Compact 2. 5-2.7 GHz two element MIMO antenna system for modern USB dongle," in Proceeding of the 6th European Conference on Antennas and Propagation (EuCAP '12), pp. 1955-1959, Prague, Czech Republic, March 2012.

[12] S. Blanch, J. Romeu, and I. Corbella, "Exact representation of antenna system diversity performance from input parameter description," Electronics Letters, vol. 39, no. 9, pp. 705-707, 2003.

[13] B. S. Yarman, Design of Ultra Wideband Antenna Matching Networks, Springer Science+Business Media B.V., 2008.

[14] P. J. Massey and K. R. Boyle, "Controlling the effects of feed cable in small antenna measurements," in Proceedings of the Antennas and Propagation Society International Symposium, vol. 2, pp. 561-564, March 2003. 

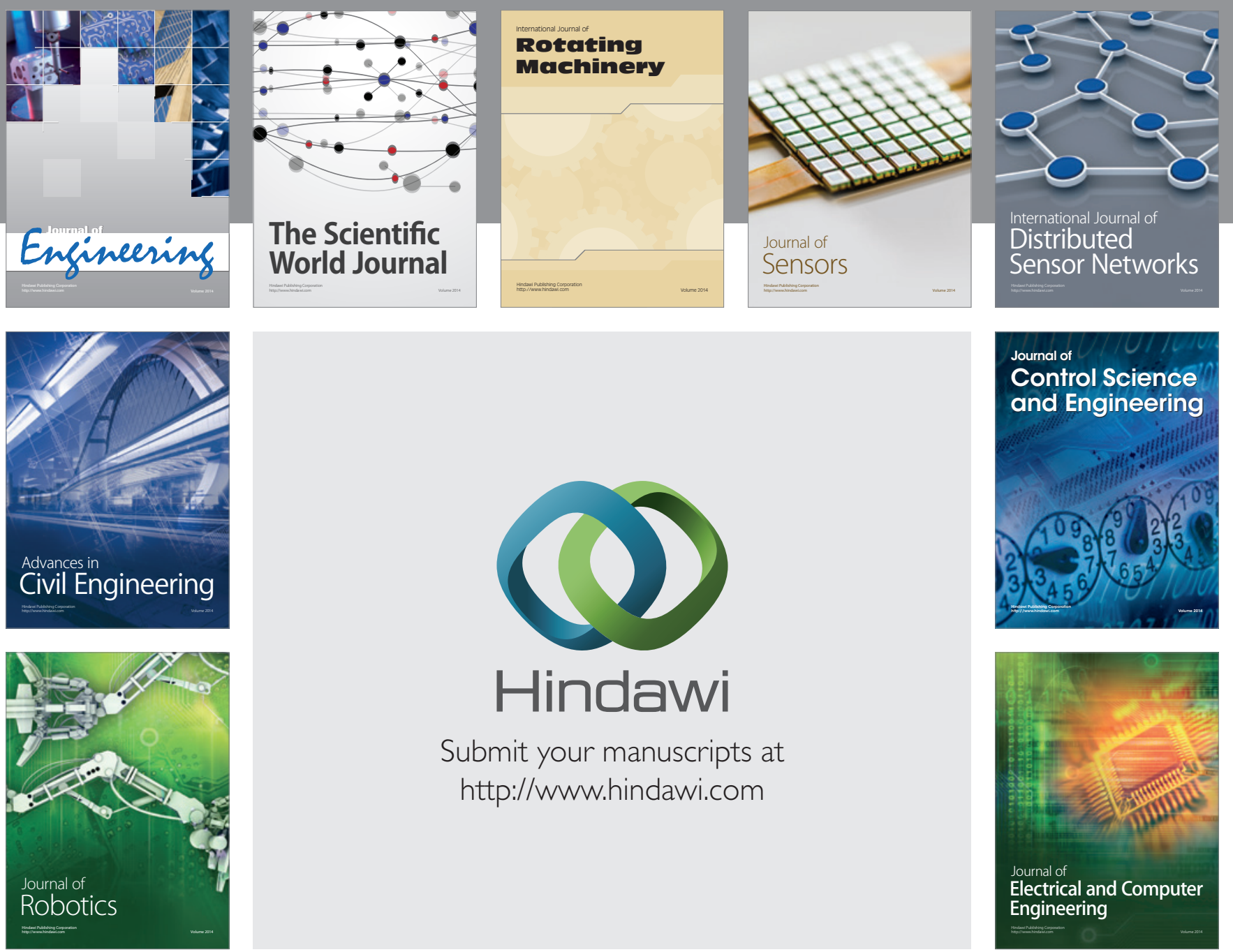

Submit your manuscripts at

http://www.hindawi.com
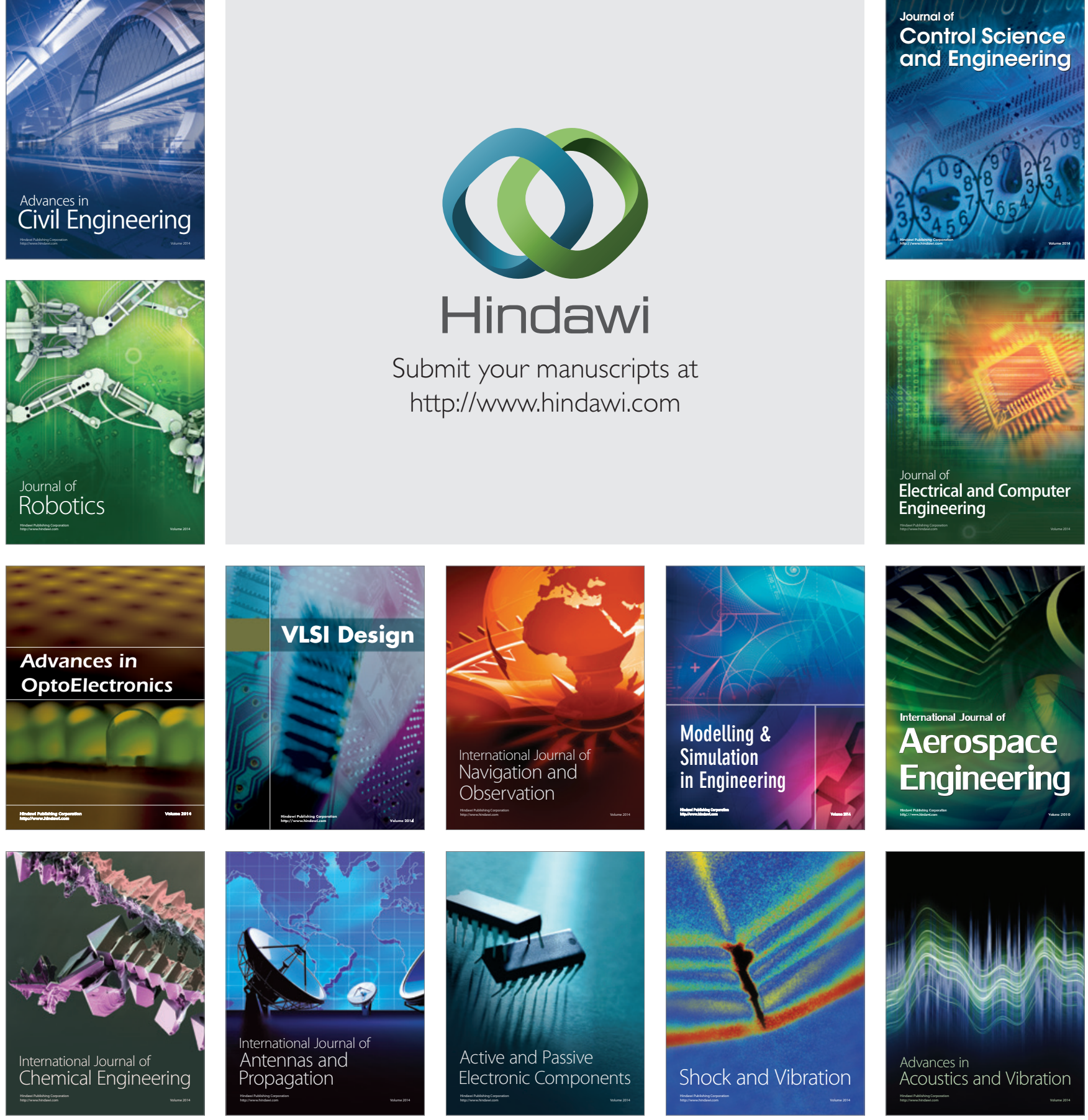